\title{
Kein erhöhtes Hirntumorrisiko durch Handys
}

\section{Entwarnung für Mobiltelefone. Fragestellung: Erhöht die Benutzung von Handys das Risiko von Hirntumoren?}



$\mathrm{D}$ erzeit nutzen etwa fünf Milliarden Menschen auf der Erde ein Mobiltelefon. Da das Gehirn dabei dessen elektromagnetischen Feldern ausgesetzt ist, gab es Befürchtungen, die häufige Benutzung könnte das Risiko von Hirntumoren erhöhen. Eine 2010 veröffentlichte große Fall-Kontroll-Studie hatte postuliert, dass bei Benutzung des Handys über einen Zeitraum von mehr als 1.640 Stunden das Risiko für Gliome erhöht sei. Langzeitstudien mit einer Beobachtungszeit von über zehn Jahren liegen allerdings bisher nicht vor.

In eine nationale Kohortenstudie wurden alle über 30-Jährigen in Dänemark aufgenommen und danach stratifiziert, ob sie vor 1995 einen Handyvertrag hatten oder nicht. Handys wurden in Dänemark erstmals 1982 eingeführt. Endpunkt waren Tumoren des zentralen Nervensystems, die das dänische Krebsregister erfasst. Bei 358.403 Menschen und 3,8 Millionen Personenjahren traten in der Zeit zwischen 1990 und 2007 10.029 Hirntumoren auf. Wurde die Analyse auf Menschen beschränkt, die mehr als 13 Jahre lang ein Handy benutzt hatten, war das Risiko eines Hirntumors nicht erhöht. Es ergab sich kein Hinweis, dass die Benutzung eines Handys mit der anatomischen Lokalisation eines Tumors in Beziehung stand.
Fazit: In einer großen nationalen Kohortenstudie fand sich kein Anhaltspunkt dafür, dass die Benutzung von Handys mit einer erhöhten Rate an Hirntumoren einhergeht.

Prof. Hans-Christoph Diener

Frei $P$ et al. Use of mobile phones and risk of brain tumours: update of Danish cohort study. BMJ. 2011;343:d6387.

Kommentar von Prof. Diener: Fall-Kontroll-Studien sind kritisch, da sie bestimmte Eigenschaften der Probanden und Risikofaktoren nicht zuverlässig erfassen. Schließen sie allerdings die Population ganzer Länder ein, werden die Aussagen deutlich zuverlässiger. Bereits die ersten Daten der dänischen Studie hatten 2002 keinen Zusammenhang zwischen Handy-Benutzung und Hirntumoren, insbesondere Gliomen und Meningeomen, gezeigt. Dies wurde jetzt repliziert. Leider hat diese für Mobiltelefonierer sehr beruhigende Studie deutlich weniger Widerhall in der Presse gefunden, als die früher publizierten kleinen Studien, die ein erhöhtes Hirntumorrisiko durch Mobiltelefone postuliert hatten. Wie üblich in unserer Presse: Nur schlechte Nachrichten sind gute Nachrichten.

Prof. Dr. med. Hans Christoph Diener

Klinik für Neurologie,

Universitätsklinikum Essen

hans.diener@uni-duisburg-essen.de 